\title{
Vessies neurologiques : les enjeux de suivi
}

\section{Neurogenic bladder: the importance of follow-up}

\section{G. Amarenco}

Reçu le 19 mars 2010 ; accepté le 19 mars 2010

(C) Springer-Verlag France 2010

Les troubles vésicosphinctériens des maladies du système nerveux posent parfois un problème diagnostique lorsqu'ils sont révélateurs de l'affection neurologique, posent souvent un problème physiopathologique important à résoudre en raison des enjeux thérapeutiques et, enfin, posent toujours celui du pronostic. C'est pour ces deux dernières raisons que le suivi des vessies neurogènes est essentiel dans la prise en charge de ces patients.

Ce suivi fait appel aux données cliniques, urodynamiques, biologiques et radiologiques.

Il n'est pas stéréotypé mais dépend du type de l'affection causale (centrale ou périphérique, médullaire ou encéphalique), de la typologie urodynamique (détrusor hyper- ou hypoactif, dyssynergie vésicosphinctérienne ou incompétence sphinctérienne), du mode évolutif de la lésion neurologique (affection stabilisée ou évolutive) et de sa diffusion (lésions disséminées ou focalisées), de la réponse thérapeutique et, enfin, des facteurs humains et environnementaux qui vont toujours moduler le mode et la fréquence de la surveillance.

Rien n'est plus dissemblable dans le suivi qu'une vessie hyperactive d'une paraplégie haute avec contractions désinhibées précoces, régime de pressions constamment trop élevées, miction incomplète à forte pression par dyssynergie vésicosphinctérienne ou qu'une vessie hypoactive d'une neuropathie périphérique responsable d'une simple dysurie. La première nécessitera des bilans urodynamiques (cystomanométrie) et radiologiques (échographie, UCRM) rapprochés pour évaluer l'impact clinique et paraclinique des thérapeutiques (anticholinergiques, alphabloquants) afin de ne pas

\footnotetext{
G. Amarenco $(\bowtie)$

Service de rééducation neurologique et d'explorations périnéales, unité Inserm U731, hôpital Rothschild, APHP,

33, boulevard Picpus, F-75012 Paris, France

e-mail : gerard.amarenco@tnn.aphp.fr
}

Public/Intérêt : Chercheurs/Faible. Cliniciens/Indispensable. Enseignants/Indispensable. Étudiants/Indispensable. Autres professions de santé/Indispensable. Cadres santé publique/Indispensable. différer des indications spécifiques (toxine botulique intradétrusorienne, entérocystoplastie) en raison du risque uronéphrologique majeur. La seconde n'imposera qu'une simple surveillance fondée essentiellement sur la clinique et sur les modifications éventuelles de la symptomatologie.

Nous disposons désormais de recommandations de suivi au cours des pathologies neurologiques susceptibles de déterminer de telles complications (lésions médullaires, scléroses en plaques) grâce au travail collectif du GENULF (Groupe européen de neuro-urologie de langue française).

Mais il existe des cas plus difficiles comme les lésions a priori pures de la queue-de-cheval avec leur vessie flasque aréactive sans risque particulier, bien contrôlées par l'autosondage, mais qui possèdent un risque évolutif à bas bruit d'hyperactivité majeure du détrusor secondaire à une lésion du cône terminal qui ne s'exprime que tardivement et qui, méconnue, peut engendrer de redoutables complications vésicorénales. La surveillance cystomanométrique régulière (tous les six mois) avec des tests à l'eau glacée permet de préjuger d'un réveil d'automatisme et de prendre les mesures correctives qui s'imposent.

Cela n'est qu'un exemple mais qui souligne la nécessaire connaissance des pathologies de leur mode évolutif et partant de leur surveillance. Cette connaissance qui devrait être partagée par le plus grand nombre n'est souvent le fait que des centres de références disposant de tout l'arsenal diagnostique et thérapeutique indispensable à la prise en charge.

Mais le suivi des vessies neurologiques n'est pas exclusivement celui du détrusor et de ses pressions. C'est aussi celui de l'homme paraplégique, de la femme sclérosée en plaques ; celui de l'individu ; celui qui souffre ; celui qui subit. Celui que l'on contraint à un traitement oral pas toujours bien supporté, pas toujours bien expliqué. Celui que l'on astreint à ces autosondages que nous, thérapeutes, tentons de démystifier au patient, mais qu'il redoute parfois, qu'il gêne souvent et qu'il considère toujours comme le témoin de son fonctionnement urinaire pour toujours défaillant. 
Cette dimension psychologique du suivi, l'appréciation des réticences du patient aux soins, le dépistage des causes de mauvaise compliance, l'évaluation du risque d'abandon des soins (autosondages, anticholinergiques) ou pire d'un suivi considéré comme devenu inutile ou psychologiquement difficile à assumer, est toujours fondamental à appréhender, à anticiper.

$\mathrm{Ne}$ nous contentons pas dans notre relation aux patients de distribuer des notices d'information et de leur fournir les statistiques des résultats des derniers traitements en vogue et des risques qu'ils encourent s'ils ne prêtent pas de bonne grâce à notre point de vue, à nos décisions, à nos narcissiques désirs...

Écoutons-les aussi...

Écoutons-les surtout...

Conflit d'intérêt : aucun. 